\title{
Quantum simulation of the Hubbard model: The attractive route
}

\author{
A. F. Ho, ${ }^{1}$ M. A. Cazalilla, ${ }^{2,3}$ and T. Giamarchi ${ }^{4}$ \\ ${ }^{1}$ Department of Physics, Royal Holloway, University of London, Egham, Surrey TW20 OEX, United Kingdom \\ ${ }^{2}$ Centro de Física de Materiales (CFM), Centro Mixto CSIC-UPV/EHU, Edificio Korta, \\ Avenida de Tolosa 72, 20018 San Sebastián, Spain \\ ${ }^{3}$ Donostia International Physics Center (DIPC), Manuel de Lardizábal 4, 20018 San Sebastián, Spain \\ ${ }^{4}$ DPMC-MaNEP, University of Geneva, 24 Quai Ernest-Ansermet, CH-1211 Geneva 4, Switzerland
}

(Received 23 December 2008; published 19 March 2009)

\begin{abstract}
We study the conditions under which, using a canonical transformation, the phases sought after for the repulsive Hubbard model, namely, a Mott insulator in the paramagnetic and antiferromagnetic phases, and a putative $d$-wave superfluid can be deduced from observations in an optical lattice loaded with a spinimbalanced ultracold Fermi gas with attractive interactions, thus realizing the attractive Hubbard model. We argue that the Mott insulator and antiferromagnetic phase of the repulsive Hubbard model are easier to observe in the attractive Hubbard mode as a band insulator of Cooper pairs and superfluid phase, respectively. The putative $d$-wave superfluid phase of the repulsive Hubbard model doped away from half filling is related to a $d$-wave antiferromagnetic phase for the attractive Hubbard model. We discuss the advantages of this approach to "quantum simulate" the Hubbard model in an optical lattice over the simulation of the doped Hubbard model in the repulsive regime. We also point out a number of technical difficulties of the proposed approach and, in some cases, suggest possible solutions.
\end{abstract}

DOI: 10.1103/PhysRevA.79.033620

PACS number(s): 67.85.Lm, 03.75.Ss, 71.10.Fd

\section{INTRODUCTION}

Understanding the phase diagram of the two-dimensional (2D) single-band Hubbard model is considered by many as the "ultimate goal" of the theory of strongly correlated systems. In the most interesting regime, this model describes a system of spin- $-\frac{1}{2}$ (i.e., two species of) fermions hopping on a 2D square lattice with repulsive (on-site) interactions and average lattice filling of less than one fermion per site. This model has been proposed as the minimal model that explains the observation of $d$-wave superconductivity with fairly high critical temperature in the doped cuprate materials [1] (for a review on doped Mott insulator, see [2]). At half filling, where only one particle per site is allowed, it is by now rather well established that the model is a Mott insulator, which at low temperatures (below a characteristic scale, the Néel temperature $\left.T_{\text {Néel }}\right)$ orders antiferromagnetically. Away from half filling, the nature of the ground state is a subject of heavy debate. One of the most challenging open issues is whether the Hubbard model on a 2D square lattice would support a $d$-wave superconducting phase at a (relatively) high temperature. The fact that the Hubbard model can support such an instability has been theoretically proven in double-chain systems coupled by hopping (known as two-leg Hubbard ladders) [3-6]. However, whether this result extends to the 2D model consisting of an infinite number of coupled chains is still extremely controversial $[2,7,8]$. At present, neither analytical nor numerical studies are able to settle the issue.

Due to the spectacular advances in the optical manipulation of ultracold atomic gases, one very promising route for studying the low-temperature phases of the Hubbard model has opened up recently [9]. Indeed, ultracold Fermi gases loaded into an optical lattice can be regarded as almost ideal quantum simulators of the Hubbard model, where indepen- dent control of the hopping amplitude $t$ and the on-site interaction energy $U$ are both experimentally available. Exploiting this fact, the Mott insulating phase of the Hubbard model has recently been demonstrated in a three-dimensional (3D) cubic optical lattice (where the center is at half filling) by several experimental groups $[10,11]$. Many other groups are currently engaged in similar experimental endeavors [12], with the main focus on realizing the repulsive Hubbard model on a 2D square lattice away from half filling, namely, the regime where $d$-wave superfluidity (SF) is speculated to exist. However, one of the main problems that lie ahead in this program has to do with the currently accessible temperatures for the Fermi gases in optical lattices. At present, these temperatures (on the order of a few tenths of the Fermi energy of a noninteracting gas of similar average density) still largely exceed the Néel temperature $\left(T_{\text {Néel }}\right)$, thereby washing out any antiferromagnetic (AFM) order in the half-filled system [13].

However, there are other problems with the present approach to simulate the repulsive Hubbard model, which seem not to have received so much attention thus far. One of the most remarkable ones is the difficulty of doping away from half filling a Fermi gas loaded in an optical lattice. In this case, the situation is very different from doping in solids, mainly because of two reasons: (i) the existence of an overall harmonic trapping potential superimposed on the optical lattice potential that makes the system inhomogeneous and tends to favor the maximum site occupancy (i.e., two fermions per site) near the center of the trap. As recently demonstrated experimentally $[10,11]$, for small number of particles this tendency can be balanced by the onsite repulsion energy, which yields a Mott insulator near the center surrounded by a "metallic" region where the density of holes is nonuniform. (ii) Although the number of available lattice sites can be controlled with accuracy, the total number of atoms is still hard to measure accurately and it is also subject to variations 
from shot to shot that are inherent to the preparation process [14].

Another source of problems has to do with the need to independently control the on-site interaction $U$ and the hopping amplitude $t$. As the Néel temperature $\left(T_{\text {Néel }}\right)$ (below which the system orders antiferromagnetically, and, upon doping, the putative $d$-wave superfluid may appear) scales as $T_{\text {Néel }} \sim t^{2} / U$, and thus rapidly decreases if the ratio $t / U$ is made very small by increasing the optical lattice depth, it is desirable to have independent control of both $t$ and $U$. In order to achieve this, the $s$-wave scattering length $a_{s}$ ( $U$ $\propto a_{s}$, roughly speaking) that characterizes the strength and sign of the atom-atom interaction must be tuned toward a Feshbach resonance where $a_{s} \rightarrow \pm \infty$. Since the current interest is in realizing a Hubbard model with repulsive interactions, the side of the Feshbach resonance where the atomatom interaction is repulsive (i.e., $a_{s}>0$ ) must be used (see, however, Sec. III for further remarks). On this side of the resonance, there is a weakly bound molecular bound state [15], with which the atoms in the continuum have a sizable overlap near the resonance. Thus, at sufficiently low temperatures, Feshbach molecules form resulting from three atom collisions [15]. A collision of one of these molecules with a third atom can cause the molecule to make a transition into a more bound molecular vibrational state. The released energy is taken away by the colliding atom, which therefore causes undesirable heating of the system. Also, the presence of these molecules is not accounted for by the single-band Hubbard model, which is the goal of the quantum simulation. Furthermore, as the scattering length increases, $U$ also increases and become of the order of the separation between Bloch bands, thus leading to the breakdown of the singleband approximation [16-18].

In this paper, we propose to explore a different route to simulate the Hubbard model in a regime where the on-site interaction is attractive. Theoretically, the attractive and repulsive regimes are related by a transformation that is well known in the literature of the Hubbard model and it is, for completeness, reviewed in Sec. II. More recently, in the context of cold atomic gases, this transformation has been used by Moreo and Scalapino [19] who pointed out the connection between the Fulde-Ferrel-Larkin-Ovchinikov state in the attractive Hubbard model and a state with stripes in the repulsive model. These authors also briefly considered the relationship between the $d$-wave superfluid order parameter for the repulsive model and a $d$-wave antiferromagnetic order in the attractive case. For the one-dimensional Hubbard model (note, however, that there is no $d$-wave superfluid phase in this case), the transformation was also used in Ref. [20] in an analysis of the noise correlations of the attractive Hubbard model with spin imbalance. Moreover, the physics of the attractive Hubbard model has also attracted much interest by itself [21], especially in recent times and in connection with cold atomic gases and the physics of the BEC to Bardeen Cooper Schrieffer (BCS) crossover [22].

In this paper, we explore in depth the possibilities offered by the attractive model to understand the physics of the repulsive Hubbard model. We pay special attention to the effects of the trapping potential as well as the peculiarities of the physical realization of the negative- $U$ Hubbard model in optical lattices. We thus argue that the attractive regime presents a number of advantages for the quantum simulation of the Hubbard model in an optical lattice. We also discuss how the (negative- $U$ ) equivalent phases of the paramagnetic Mott insulator, the antiferromagnetically ordered Mott insulator, and the putative $d$-wave superfluid may be observed.

We would like to emphasize that one of the main advantages of the route suggested here is that the equivalence of doping away from half filling the optical lattice system in the repulsive regime can be achieved by creating a spinimbalanced gas. Fermi gases with spin-imbalanced populations are nowadays routinely created in the laboratory [23], and the magnetization (which is fixed for the duration of the experiment by the preparation method) can be controlled to within a few percents accuracy. Other advantages will be discussed further below. However, our approach does not solve the problem of how to achieve lower temperatures for the fermions on the lattice [13].

The outline of this paper is as follows. In Sec. II we discuss the transformation that formally maps the repulsive Hubbard model into an attractive one. We also define the two attractive Hubbard models we shall be concerned with in this paper, together with their corresponding repulsive models. We also describe how various physical operators and order parameters are affected by the mapping. Some important caveats concerning the realization of the attractive Hubbard model are discussed in Sec. III. In Sec. IV we discuss the equivalent state of the paramagnetic phase of the Mott insulator (as well as possible ways of detecting it), whereas in Sec. $\mathrm{V}$ we do the same for the equivalent state of the antiferromagnetically ordered Mott insulator. The effect of doping with holes, which, as mentioned above, amounts to a spin-imbalanced situation in the attractive case, is analyzed in Sec. VI. Finally, in Sec. VII we offer the conclusions of the present work as well as mentioning some open problems.

\section{HUBBARD MODEL AND THE PARTICLE-HOLE TRANSFORMATION}

The Hamiltonian of the single-band Hubbard model reads

$$
H=-t \sum_{\langle i j\rangle \sigma} c_{i \sigma}^{\dagger} c_{j \sigma}+U \sum_{i}\left(n_{i \uparrow}-\frac{1}{2}\right)\left(n_{i \downarrow}-\frac{1}{2}\right)+H_{\mathrm{ext}},
$$

where $t$ is the hopping amplitude and $U$ is the one-site interaction. We consider here only the case where the sites $i$ of the lattice constitute a hypercubic lattice (square in two dimensions and cubic in three dimensions); $\langle i, j\rangle$ in the hopping term means that the sum runs over nearest-neighbor sites only. In the above equation, $n_{i \sigma}=c_{i \sigma}^{\dagger} c_{i \sigma}$ is the occupancy of spin $\sigma=\uparrow, \downarrow$ fermions at the $i$ th site. For simplicity and unless otherwise stated, we will refer in the following to the two-dimensional case, and thus to a square lattice. All our results are straightforwardly generalizable to the case of any hypercubic lattice.

We have denoted as $H_{\text {ext }}$ all the external fields such as chemical and trap potentials as well as an external Zeeman (magnetic) field that act upon the system [24]. Their effects will be discussed below. In the grand canonical ensemble, 
TABLE I. Transformation between phases (and their associated order parameters) of the Hubbard model for $U>0$ and $U<0$ under the canonical transformation of Eq. (4) [cf. Eq. (6) in momentum space]. In the expressions below, $\phi_{k}^{(d)}=\left(\cos k_{x}-\cos k_{y}\right)$ is the $d_{x^{2}-y^{2}}$ lattice form factor.

\begin{tabular}{lc}
\hline \hline \multicolumn{1}{c}{$U>0$} & $U<0$ \\
\hline Paramagnetic Mott insulator (MI, $\left.n_{i}=1\right)$ & Disordered lattice with $n_{i}=0$ or $n_{i}=2$ \\
$s$-wave AFM MI $(z) M_{Q}^{(s) z}=\Sigma_{k} \Sigma_{\sigma} \sigma\left\langle c_{k \sigma}^{\dagger} c_{k+Q \sigma}\right\rangle$ & $s \mathrm{CDW}$ insulator $\Delta_{Q}^{(s) \mathrm{CDW}}=\Sigma_{k} \Sigma_{\sigma}\left\langle c_{k \sigma}^{\dagger} c_{k+Q \sigma}\right\rangle$ \\
$s$-wave AFM MI $(x, y) M_{Q}^{(s)-}=\Sigma_{k}\left\langle c_{k+Q \downarrow}^{\dagger} c_{k \uparrow}\right\rangle$ & $s$-wave superfluid $\Delta^{(s)}=\Sigma_{k}\left\langle c_{-k \downarrow} c_{k \uparrow}\right\rangle$ \\
$d$-wave superfluid (?) $\Delta^{(d)}=\Sigma_{k} \phi_{k}^{(d)}\left\langle c_{-k \downarrow} c_{k \uparrow}\right\rangle$ & $d$-wave AFM metal (?) $M_{Q}^{(d)-}=\Sigma_{k} \phi_{k}^{(d)}\left\langle c_{k+Q \downarrow}^{\dagger} c_{k \uparrow}\right\rangle$ \\
\hline \hline
\end{tabular}

$$
H_{\mathrm{ext}}=-\mu N+h M,
$$

where the total number operator $N=\Sigma_{i, \sigma} n_{i \sigma}$ and the total magnetization $M=\Sigma_{i}\left(n_{i \uparrow}-n_{i \downarrow}\right)$, and $\mu$ along with $h$ is determined by the condition that averages of $N$ and $M$ over the grand canonical ensemble yield the experimentally observed values [25]. However, cold atomic gases are prepared in eigenstates of both $N$ and $M$, and therefore the relevant ensemble is canonical instead of grand canonical. Although it is important to keep this distinction in mind, we expect that for sufficiently large $N$, the results of both ensembles coincide, and thus we shall use the grand canonical for the calculation of the experimental signatures of the different phases to be described below in Sec. VI.

The Hamiltonian of Eq. (1) has been written in a form such that for a uniform system the ground state will have exactly one particle per site, at any temperature, for $U>0$ and $H_{\text {ext }}=0$. However, note that in real experiments cold atomic gases are harmonically trapped. Therefore, the most general form of $H_{\text {ext }}$ reads

$$
H_{\mathrm{ext}}=\sum_{i}\left(\epsilon_{i}-\mu\right)\left(n_{i \uparrow}+n_{i \downarrow}-1\right)-\sum_{i} h_{i}\left(n_{i \uparrow}-n_{i \downarrow}\right),
$$

where $\epsilon_{i}$ is the shift in the local chemical potential caused by the trap. We have added an unimportant constant to the total energy $\left[=\sum_{i}\left(\epsilon_{i}-\mu\right)\right]$, which will become convenient further below. In current optical lattice experiments, we have $\epsilon_{i}$ $=\frac{1}{2} \epsilon_{0} i^{2}$, but more general forms of the trap may become available in the future. In the case relevant to experiments, the Zeeman field $h_{i}=h$ is uniform (and it is used to adjust the total magnetization in the grand canonical ensemble). However, in Eq. (3) we have assumed it to be site dependent for further convenience.

We next note that, formally, the sign of the interaction term [the term $\propto U$ in Eq. (1)] can be changed by means of the following (particle-hole) transformation on a bipartite lattice such as the 2D square lattice:

$$
\begin{aligned}
c_{i \downarrow}=c_{i_{x} i_{y} \downarrow} & \leftrightarrow(-1)^{i_{x}+i_{y}} c_{i \downarrow}^{\dagger}, \\
c_{i \uparrow} & \leftrightarrow c_{i \uparrow} .
\end{aligned}
$$

Note that the transformation leaves the operators of the spin $\uparrow$ fermions unchanged. However, it affects the spin $\downarrow$ occupation operator, $n_{i \downarrow} \leftrightarrow 1-n_{i \downarrow}$, and thus the sign of the interaction term changes $(U \leftrightarrow-U)$ while hopping term is left invariant [the minus sign in the right-hand side of Eq. (4) takes care of this]. However, it will be important for the discussion that follows that the transformation exchanges the roles of $h_{i}$ and $\left(\epsilon_{i}-\mu\right)$ in Eq. (3). Mathematically,

$$
H_{\mathrm{ext}} \rightarrow H_{\mathrm{ext}}^{\prime}=\sum_{i}\left(\epsilon_{i}-\mu\right)\left(n_{i \uparrow}-n_{i \downarrow}\right)-\sum_{i} h_{i}\left(n_{i \uparrow}+n_{i \downarrow}-1\right) .
$$

If we insist in using the point of view of the canonical ensemble, transformation (4) implies that $N \rightarrow N^{\prime}=M+\mathcal{N}$ and $M \rightarrow M^{\prime}=N-\mathcal{N}$, where $\mathcal{N}$ is the total number of lattice sites. Thus, if we consider an unmagnetized (i.e., $M=0$ ) system where $N=(1+x) \mathcal{N}$ ( $x$ being the doping, where $x=0$ corresponds to half filling in the uniform case), we have that $N^{\prime}$ $=\mathcal{N}$ and $M^{\prime}=x \mathcal{N}$. In words, the doped lattice at $U>0$ away from half filling maps onto a $U<0$ system at finite magnetization. We emphasize that, as discussed below, the details of the order that the system develops depend not just on usual factors such as the temperature and strength of the trapping and lattice potential, but are also constrained by these globally conserved quantities.

It is also convenient to recall that, in momentum space, the transformation of Eq. (4) becomes

$$
c_{k \downarrow} \leftrightarrow c_{k+Q \downarrow}^{\dagger}, \quad c_{k \uparrow} \leftrightarrow c_{k \uparrow},
$$

where $Q=(\pi / a, \pi / a)$ is the nesting vector with $a$ as the lattice spacing. This expression can be used to obtain the way the different order parameters and the corresponding phases transform between the $U>0$ and the $U<0$ cases. This is shown in Table I. The way the transformation affects the different phases expected for the Hubbard model is also illustrated in Fig. 1.

Finally, for the sake of clarity, we will spell out in what follows the two physically different attractive Hubbard models considered in this paper, as well as the repulsive Hubbard models onto which, via the particle-hole transformation (4), they are mapped. The first attractive Hubbard model (from here on called model A1) has the following Hamiltonian (in the grand canonical ensemble):

$$
\begin{aligned}
H_{\mathrm{A} 1}= & -t \sum_{\langle i j\rangle \sigma} c_{i \sigma}^{\dagger} c_{j \sigma}-|U| \sum_{i}\left(n_{i \uparrow}-\frac{1}{2}\right)\left(n_{i \downarrow}-\frac{1}{2}\right) \\
& +\sum_{i}\left(\epsilon_{i}-\mu\right)\left(n_{i \uparrow}+n_{i \downarrow}-1\right)-h \sum_{i}\left(n_{i \uparrow}-n_{i \downarrow}\right) .
\end{aligned}
$$

This is the Hamiltonian that describes cold atomic systems trapped in an optical lattice, with the overall harmonic trapping potential $\epsilon_{i}=\frac{1}{2} \epsilon_{0} i^{2}$, and a uniform Zeeman field that can 


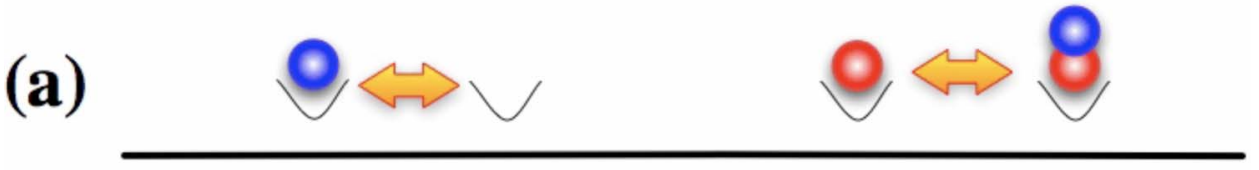

\section{REPULSIVE HUBBARD MODEL ATTRACTIVE HUBBARD MODEL}
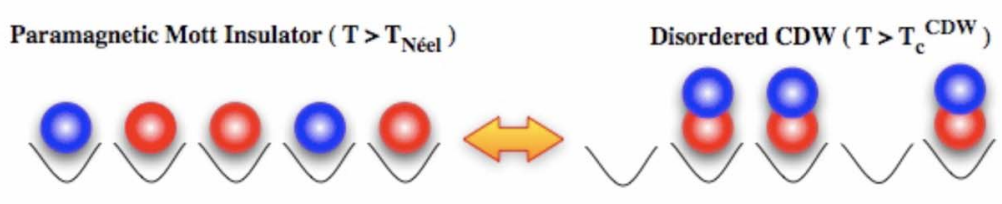

(b)

\begin{abstract}
Anti-ferromagnetic Mott Insulator $\left(T<T_{\text {Néel }}\right)$
\end{abstract}
Ordered CDW $\left(\mathrm{T}<\mathrm{T}_{\mathrm{c}} \mathrm{CDW}\right)$

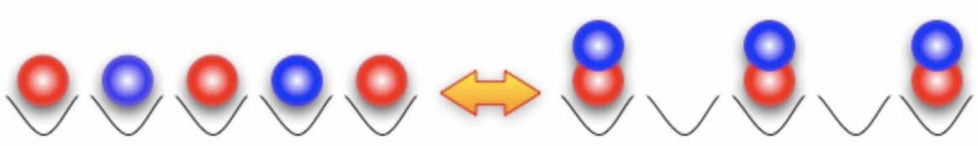

Doped Anti-ferromagnetic Mott Insulator (d-wave superfluid)

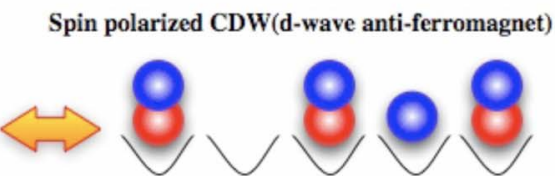

FIG. 1. (Color online) Schematic of how the transformation of Eq. (4) works. In (a) we sketch how it acts on a single site (e.g., red stands for spin up and blue for spin down) and in (b) the way it transforms different types of states on a uniform optical lattice. In addition to the phases depicted above, an antiferromagnetic state at $U>0$ ordered along the $x$ or $y$ direction corresponds to a superfluid phase of fermion pairs. The bottom diagram illustrates one of the main points of this paper, namely, that doping the attractive Hubbard model corresponds to introducing spin imbalance in the repulsive Hubbard model. be viewed as a knob to tune the spin imbalance (see Sec. III). In this paper, we argue that simulating this Hamiltonian has a number of advantages over current attempts at simulating the repulsive Hubbard model in the presence of the harmonic trap (from here on called model R2), whose Hamiltonian reads

$$
\begin{aligned}
H_{\mathrm{R} 2}= & -t \sum_{\langle i j\rangle \sigma} c_{i \sigma}^{\dagger} c_{j \sigma}+|U| \sum_{i}\left(n_{i \uparrow}-\frac{1}{2}\right)\left(n_{i \downarrow}-\frac{1}{2}\right) \\
& +\sum_{i}\left(\epsilon_{i}-\mu\right)\left(n_{i \uparrow}+n_{i \downarrow}-1\right) .
\end{aligned}
$$

The other attractive Hubbard model (from here on called model A2) is described by the following Hamiltonian:

$$
\begin{aligned}
H_{\mathrm{A} 2}= & -t \sum_{\langle i j\rangle \sigma} c_{i \sigma}^{\dagger} c_{j \sigma}-|U| \sum_{i}\left(n_{i \uparrow}-\frac{1}{2}\right)\left(n_{i \downarrow}-\frac{1}{2}\right) \\
& +\sum_{i}\left(\epsilon_{i}-\mu\right)\left(n_{i \uparrow}-n_{i \downarrow}\right) .
\end{aligned}
$$

This is the Hamiltonian that can be obtained from the repulsive model R2 [Eq. (8)] via the particle-hole transformation of Eq. (4). Model A2 has an inhomogeneous Zeeman field stemming (via the transformation) from the trapping potential of model R2. In this paper, model A2 is used to help us understand, e.g., the coexistence of phases in the current experimental regime of the repulsive Hubbard model (R2). Note that the attractive model A1 that we are advocating does not map onto the currently studied repulsive Hubbard model R2, but instead onto the repulsive Hubbard model in an inhomogeneous Zeeman field (called model R1 here),

$$
\begin{aligned}
H_{\mathrm{R} 1}= & -t \sum_{\langle i j\rangle \sigma} c_{i \sigma}^{\dagger} c_{j \sigma}+|U| \sum_{i}\left(n_{i \uparrow}-\frac{1}{2}\right)\left(n_{i \downarrow}-\frac{1}{2}\right) \\
& +\sum_{i}\left(\epsilon_{i}-\mu\right)\left(n_{i \uparrow}-n_{i \downarrow}\right)-h \sum_{i}\left(n_{i \uparrow}+n_{i \downarrow}-1\right) .
\end{aligned}
$$

Thus, to summarize, using the mathematical transformation of Eq. (4), we can relate the physics of the attractive Hubbard models to that of the repulsive ones. In particular the observation of one particular phase [e.g., a $d$-wave antiferromagnetic (AFM) phase] at $U<0$ would directly imply the existence of the corresponding phase at $U>0$ (the putative $d$-wave superfluid phase). Below we shall see that the realization of some of these phases in the attractive regime requires sometimes less stringent conditions than the corresponding ones in the repulsive regime. Furthermore, as described above, the exchange of roles of the Zeeman field and the chemical potential terms effected by the transformation implies that we can simulate the doping of the Mott insulator by creating a system with a finite magnetization (i.e., a spinimbalanced system). Also, even if the temperatures that can be achieved in current experiments do not allow for the investigation of the low-temperature ordered states, we may expect that, by the attractive route, some useful insights can be gained into other controversial issues for the high- $T_{c}$ community, such as the nature of the normal state of the $2 \mathrm{D}$ repulsive Hubbard model away from half filling.

\section{REALIZATION OF THE ATTRACTIVE HUBBARD MODEL}

In principle, since the onsite interaction energy $U$ is $n a$ ively proportional to the atomic scattering length in free space [18], $a_{s}$, the $U<0$ regime can be accessed by sweeping 
the magnetic field to the side of an interspecies Feshbach resonance [15] where $a_{s}<0$. In the literature of the BCS to $\mathrm{BEC}$ crossover $[15,23,26]$, this side is known as the "BCS side" of the resonance.

However, the above point of view entirely neglects the subtleties of the scattering problem on a lattice potential, as it turns out that $U$ is not a linear function of $a_{s}$, in the general case [27-29]. The details of the dependence of the zero momentum scattering amplitude, $f_{0}\left(a_{s}\right)$ (and $U \propto f_{0}$ ), on the atomic scattering length $a_{s}$ are determined by the dimensionality and other parameters of the lattice [27-29]. However, all these results share one common feature, namely, the existence of a particular length scale $l_{*}>0$, such that for $a_{s}$ $=-l_{*}$ the scattering amplitude $f_{0}$ exhibits a (geometric) resonance [27-29]: $f_{0} \propto a_{s} /\left(1+a_{s} / l_{*}\right)$. Indeed, the resonance can be approached either by changing the lattice parameters or by changing $a_{s}$ through a Feshbach resonance. We shall focus on the latter case here. To realize the attractive Hubbard model $U<0$, we require tuning the scattering length to the attractive side $\left(a_{s}<0\right)$ but such that $\left|a_{s}\right|<l_{*}$. As we approach the Feshbach resonance and $f_{0}$ diverges to $-\infty$, crossing the geometric resonance beyond $l_{*}$ leads to the interaction becoming effectively repulsive and, as a consequence, close to the geometric resonance, a weakly bound state appears. The existence of this bound state has been discussed in the literature $[27,28]$, and if the temperature is sufficiently low (compared to the binding energy of the bound state), many bound states will be created even if the effective interaction between the atoms in the lattice is repulsive. This regime is clearly not described by the repulsive Hubbard model because it does not take into account the bound states. The situation is similar (although probably less harmful for the system [27]) to the one encountered as $a_{s} \rightarrow+\infty$, on the socalled BEC side of the Feshbach resonance. In this regime, the scattering amplitude corresponds to that of a repulsive effective interaction, which may lead us to think that the system is described by the repulsive Hubbard model, except crucially, for the existence of the lattice molecular bound states. But indeed, as described in Sec. I, the existence of Feshbach molecules [15] leads to inelastic losses.

To summarize, the attractive regime can be reached by making the scattering length $a_{s}$ negative, but not beyond a certain limit where, for $a_{s}=-l_{*}\left(l_{*}\right.$ depending on the lattice dimensionality and other parameters [27-29]), the scattering amplitude has a geometric resonance.

\section{ANALOG OF THE MOTT INSULATOR IN THE ATTRACTIVE REGIME}

Let us first start by looking at the $U<0$ system with a balanced population of spin-up and -down fermions [model A1, Eq. (7) with $h=0]$ and at temperatures $T$ where $|U|$ can be made such that $T<|U|$ (however, in this section $T$ is assumed to be large compared with $t^{2} /|U|$ ). Using the transformation [Eq. (4)] this corresponds to a half-filled system for $U>0$ [model R1, Eq. (10) with $h=0$ ]. This is the situation where it is known that a Mott insulator appears in model R1. For $U>0$ the existence of the Mott insulator means that the states with more than one particle per site are strongly dis- favored due to the large on-site repulsion $U$. The corresponding situation for $U<0$ is that the only allowed states for every lattice site are either zero or doubly occupied states, as shown in Fig. 1. The existence of the Mott insulator in the repulsive regime thus corresponds in the attractive regime to having all fermions form pairs [30]. For sufficiently large $|U|$ these pairs are tightly bound, which means that their existence can be probed by sending photons to photoassociate them into dimers, which are no longer trapped, and therefore can be detected as a loss of atoms from the lattice [31].

It is worth noticing that this system of pairs exhibits a pairing gap $(\sim|U|$ for large $|U|)$ to spin excitations. Therefore, a measurement of the single-particle properties, such as the single-particle spectral function which is accessible by photoemissionlike spectroscopy proposed in [32] and recently applied to ultracold Fermi gases by the JILA group [33], should be able to detect the pairing gap. However, since in model A1 the harmonic trap only couples to the atomic density [cf. Eq. (7)], it does not lead to the breaking of the pairs and, therefore, it does not take the system out of the subspace where $n_{i}=0$ or $n_{i}=2$ [which, by virtue of Eq. (4) corresponds to half-filling, i.e., $\left.n_{i}=1\right]$. Indeed, the role of the trap is to lift the large energy degeneracy (for $t=0$ ) of the states in this subspace. In other words, in the absence of the trapping potential in model A1, and since the chemical potential $\mu$ couples to the total particle number, all states with the same total number of fermions and only doubly occupied or empty sites are degenerate. The trap breaks this degeneracy and selects as the ground state of model A1 the state where all pairs uniformly occupy the lattice sites at the trap bottom. In the strong-coupling limit, this state can be regarded as a "band insulator" of the pairs (see Fig. 3).

A complementary way of arriving at the same conclusion relies on the transformation of Eq. (4). After the transformation, model A1 at $h=0$ is mapped to model R1, that is, a repulsive Hubbard model but in an inhomogeneous Zeeman field (note that $h=0$ in this model too, but in this case it couples to the total density). Although the Zeeman field affects the magnetic ordering by ferromagnetically polarizing the fermions at the center of the trap, it does not lead to doubly occupied sites, and therefore it does not affect the characteristic incompressibility of the Mott insulator, which depends on the existence of a gap $(\sim U$ for large $U)$ to all density excitations.

It is worth to contrast the situation described in previous paragraphs with the one found in current experiments, which are performed in the repulsive regime of the Hubbard model [model R2, Eq. (8)]. In such a system, one needs to adjust the chemical potential $\mu$ (that is, the number of fermions, $N$ ) in order to have a half-filled lattice with one fermion per site at the trap bottom. Otherwise, at too large $\mu / U$ (i.e., large $N)$, the system energetically prefers to pay the energy cost of having doubly occupied sites near the bottom of the trap rather than accumulating them far from the center, where the trapping energy is very large. Thus, the lattice at the center of the trap ceases to be in the Mott insulating phase, becoming a band insulator. On the other hand, for too small $\mu$ (i.e., small $N$ ), the optical lattice is not uniformly occupied by one fermion site. Testing for the existence of the Mott insulator at $U>0$ thus requires checking for the absence of doubly oc- 


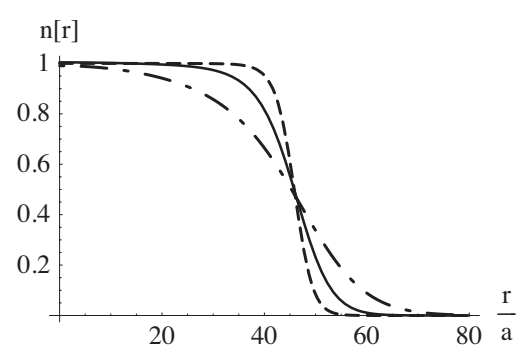

FIG. 2. Density profile for the repulsive Hubbard model in a harmonic trap (model R2) as a function of the radial distance from the trap center $r$ in units of $a$, the optical lattice spacing. Dashed line: $T / U=0.05$, solid line: $T / U=0.1$, and dotted-dashed line: $T / U=0.2$. The total number of particles is $N=6599$. The trapping energy $\epsilon_{0} / 2 U=0.0003$.

cupied sites, which has been already achieved experimentally by the Zürich and Mainz groups $[10,11]$. However, testing the absence of holes (which may appear due to thermal or quantum fluctuation, especially as $N$ or $U$ decreases) is a more difficult task. In this regard, in the attractive regime, only the absence of singly occupied states needs to be tested. Such measurement, which implies recording the spatial distribution of lattice sites with different occupations, may be accessible through spectroscopic techniques similar to those employed to observe the "wedding-cake" structure of the bosonic Mott insulator [34,35].

In Figs. 2 and 3 we show illustrative plots (in the large $|U| / t$ limit and for $T<|U|)$ of the experimentally measurable or measured density profile $n(r)=n_{\uparrow}(r)+n_{\downarrow}(r)$, both in the attractive regime with no spin imbalance (Fig. 3 for model A1 at $h=0)$ and in the repulsive regime, the half-filled lattice (Fig. 2 for model R2) [36]. We have used data similar to those in current experiments, e.g., the Zürich group [10] (see figure captions).

It is noticeable that for $U>0$ (model R2), for the given number of particles vs trap energy $\epsilon_{0}=m \omega^{2} a^{2}$ ( $m$ is the mass of a single fermion and $\omega$ is the trapping frequency), there is single occupancy in the central region, which is the Mott insulator region. At temperatures that are low compared to $U$, but higher than any second-order (exchange, etc.) processes at the energy scale $\sim t^{2} / U$ (i.e., at currently achievable temperatures), the $\uparrow$ and $\downarrow$ fermions are equally likely to be found on any site of the Mott-insulating region, as there is no magnetic ordering. The latter can only emerge as the temperature is lowered below the Néel scale $T_{\text {Néel }} \sim t^{2} / U$. As we move toward the edge of the sample, the site occupancy deviates noticeably from one fermion per site, and a metallic shell appears. Its width depends on the temperature, as well as $t, U$, and the trapping energy.

On the other hand, for the $U<0$ case of model A1 at $h$ $=0$, the center of the lattice is filled with fermion pairs resulting from the attractive on-site interaction. Thus, in model A1 (Fig. 3) the effect of temperature (in the limit where one can disregard the second-order effects due to the hopping) is to create a finite density of empty sites near the edge of the band insulator of pairs, where the entropy is concentrated. This explains the deviation of the site occupancy from $n(r)$ $=2$ observed in Fig. 3, as the distance to the center, $r$, increases. We also notice that, because the trap is not in com-

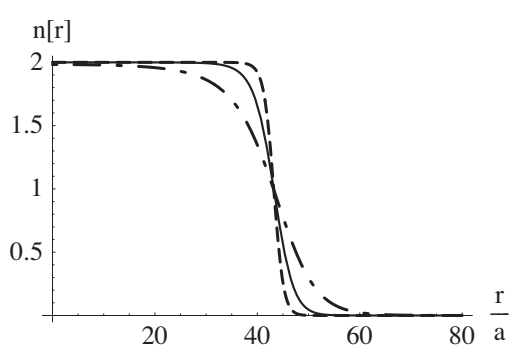

FIG. 3. Density profile for the attractive Hubbard model in a harmonic trap (model A1 at $h=0$ ) as a function of the radial distance from the trap center. Dashed line: $T / U=0.05$, solid line: $T / U=0.1$, and dotted-dashed line: $T / U=0.2$. The total number of particles is $N=11728$. The trapping energy $\epsilon_{0} / 2 U=0.0003$.

petition with the pairing gap as described above, the stability of the band insulator state is not threatened by the trap (contrary to the Mott insulator at $U>0$ ), and thus the fermion numbers in this regime $(U<0)$ can be larger than those at $U>0$, which is also illustrated by the sizes used to generate the figures. However, for model R2, the trap, as described above, tends to favor the band insulator in the middle for large enough $N$. When the total number of fermions $N$ becomes smaller than a critical value (see Sec. V), consideration of the effects of hopping will be required.

\section{ANTIFERROMAGNETIC ORDER FOR $U>0$ AND ITS ANALOG IN ATTRACTIVE CASE}

As discussed in Sec. IV, for large on-site attraction, the only two possible states for a single site are single and doubly occupied. Singly occupied sites are separated from these states by the (large) energy gap $\sim|U|$. Thus all the fermions are paired and can be regarded as hard-core bosonic entities hopping from site to site with amplitude $\sim t^{2} /|U|$. To see this, recall that, in the repulsive case [37] and ignoring for the moment the overall harmonic trapping, the low-energy effective model for the $U>0$ half-filled Hubbard model (with equal proportion of the two species) is a spin-1/2 nearestneighbor Heisenberg model $H_{\text {eff }}(U>0)=J \sum_{\langle i, j\rangle} S_{i} S_{j}$, where $J$ $=4 t^{2} /|U|$. This model transforms for $U<0$, upon applying Eq. (4), into a half-filled lattice described by the following effective Hamiltonian in terms of hard-core bosons $\left(b_{i}\right.$ $\left.=c_{i \downarrow} c_{i \uparrow}\right)$ :

$$
H_{\mathrm{eff}}(U<0)=J \sum_{\langle i, j\rangle}\left[\left(b_{i}^{\dagger} b_{i}-\frac{1}{2}\right)\left(b_{j}^{\dagger} b_{j}-\frac{1}{2}\right)-b_{i}^{\dagger} b_{j}\right],
$$

where $\langle i, j\rangle$ means that the sum runs over nearest-neighbor sites only. For the $U>0$ case, the ground state of the Heisenberg model is a ( $s$-wave) antiferromagnet ( $s$ AFM). In the absence of any terms in the Hamiltonian that distinguish spin-up and -down fermions, that is, for a spin-isotropic Hamiltonian, the staggered magnetization can point in any of the spin directions $x, y$, or $z$.

For the $U<0$ case, the resulting system [Eq. (11)] is thus a system of hard-core bosons (arising from the pairing of two different spin fermions) hopping on the lattice with the ki- 
a) $\bigotimes$ spin into paper $(-z)$
$\odot$ spin out of paper $(+z)$

b)
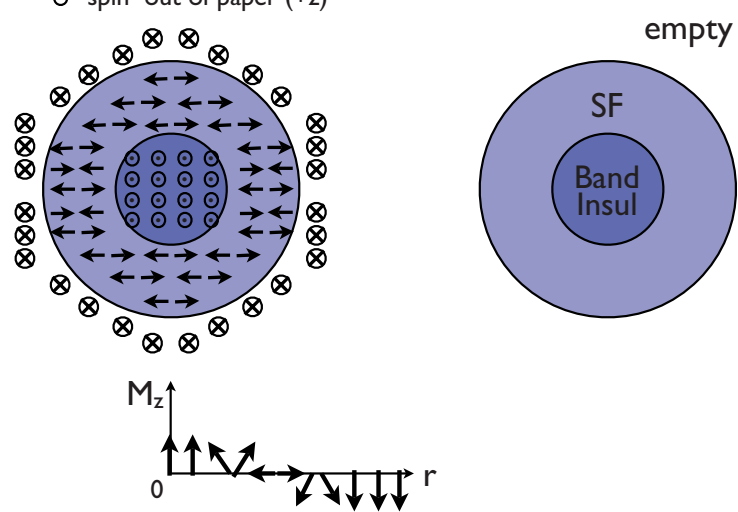

FIG. 4. (Color online) Schematic of the shell structure of (a) the half-filled repulsive Hubbard model in a site-dependent Zeeman field (model R1) and (b) the corresponding attractive model A1 state configuration in a trap.

netic energy $\sim J$ and experiencing nearest-neighbor repulsion interaction also $\sim J$. Several phases can be realized in such a system. The resulting nearest-neighbor repulsion and kinetic energy in Eq. (11) favor an alternating pattern of empty and doubly occupied states, i.e., the checkerboard state in 2D also known as the "charge-density wave" (CDW) state. This alternating pattern of empty and doubly occupied states for $U<0$ is the analog of the antiferromagnetic (along the $z$ direction) state for $U>0$ as shown in Fig. 1 and Table I. Another alternative ground state is simply an $s$-wave superfluid of these bosonic pairs $(s \mathrm{SF})$. In fact, the degeneracy for ordering along any direction $x, y, z$ of the $s$ AFM for the $U$ $>0$ case maps to a degeneracy between the CDW state and the SF state for the $U<0$ case, which one can see formally from the order-parameter mapping, using transformation (6): the checkerboard order parameter $\Delta^{\mathrm{CDW}}$ maps to the antiferromagnetic order parameter in the $z$-spin direction $M_{\text {stag }}^{z}$, while the SF order parameter $\Delta^{(s)}, \Delta^{(s)^{\dagger}}$ maps to the AFM order along,-+ direction, $M_{\mathrm{stag}}^{+}=M_{\mathrm{stag}}^{x}+i M_{\mathrm{stag}}^{y}$ and $M_{\mathrm{stag}}^{-}$ $=M_{\text {stag }}^{x}-i M_{\text {stag }}^{y}[38]$ (see Table I).

However, the presence of the harmonic trap on the attractive side leads to interesting effects. As can be seen from Eq. (3), in model A1 [Eq. (7)], the trap acts as a chemical potential for the pairs in the $U<0$ case and thus favors a completely filled center of pairs [which is just a band insulator in the center (cf. Fig. 3)] rather than either a CDW or superfluid state. In fact, in the limit where tunneling between sites is suppressed, the ground state in the trap potential is this band insulator, and only the kinetic energy and the nearestneighbor repulsion of order $J$ prevent this state to occur. Mapping back to the $U>0$ case, one sees that the trap transforms into a Zeeman field along the $z$ direction to become model R1 [Eq. (10)]. This Zeeman field will lift the degeneracy between the various magnetic states and then polarize ferromagnetically the spins rather than favor an antiferromagnetic order. This competition is summarized in Fig. 4.

This effective Zeeman field in model R1 goes from large and positive in the center of the trap to large and negative in the periphery. In the center of the trap one would thus have all the spins polarized up. This phase corresponds, via the transformation, to a pair on each site and thus to the band insulator of pairs in model A1. Whether the effective field in the center of the trap is enough to polarize fully the spin depends on the number of particles in the trap and will be discussed below. Further from the center, the Zeeman field decreases and becomes negative. One has thus in a certain radius a shell where the spins are not fully polarized. In this region the spins preserve an antiferromagnetic order in the direction perpendicular to the Zeeman field albeit with a reduced amplitude (i.e., the AFM order parameters $M_{Q}^{(s) \pm}$ are nonzero). In other words, in this shell, the system of model $\mathrm{R} 1$ thus possesses antiferromagnetic order in the $x, y$ directions in spin space. After the transformation this $x, y$ antiferromagnetic phase for $U>0$ maps for $U<0$ to an $s$-wave superfluid phase, as shown in Fig. 4. We thus see that looking for antiferromagnetism in the repulsive Hubbard model amounts to probing for superfluidity in the $U<0$ one. Then beyond a certain radius the trap prevents the pair to exist, and the system is empty (see Fig. 3). In the $U>0$ system, this corresponds to a Zeeman field that is large enough to fully polarize to down the system of spins [39].

In summary, on the $U<0$ side the trap does not really spoil the search for the analog of the antiferromagnetic phase. The corresponding phase is now a simple $s$-wave superfluid that can be probed by similar techniques that have already been used in the continuum. The extra phases induced by the trap only potentially reduce the spatial extent of the superfluid shell, but since they both correspond to insulating regions (either band insulator or empty region), they should not spoil the observation of the superfluidity. To minimize the central band insulator region for the attractive model A1 (and hence maximize the superfluid signal), the chemical potential at the trap center must not be too strong, so that when tunneling is turned on, the resulting pair hopping and pair repulsion energy scale $J$ [see Eq. (11)] can overcome the trapping potential to delocalize the band insulator. First, in the strong-coupling limit, consider the case where the whole trap consists of mostly the band insulator. We can estimate the instability of the band insulator to this scale $J$ : taking a pair at the edge of the band insulator system $\left(R=R_{2}\right)$ to a site just beyond the edge (by a lattice spacing $a$ ) costs an energy $\sim \epsilon_{0}\left(R_{2} / a\right)$, and this is to be balanced against $J=4 t^{2} /|U|$. For a large enough system, $\pi\left(R_{2} / a\right)^{2}=N / 2$, where $N / 2$ is the total number of pairs. Thus the band insulator state will become unstable when roughly $t^{2} /|U|$ $>\epsilon_{0} \sqrt{N / 2 \pi}$. However, for a full SF state in the central region of the trap, one needs to move outward more than just the pairs at the boundary of the band insulator. A similar estimation indicates that $\sqrt{N}$ in the above criterion is replaced with linear in $N$. Thus for a given trap energy $\epsilon_{0}$, there is an upper limit to total number of fermions that can be loaded into the trap. For example, using data similar to those in the ${ }^{40} \mathrm{~K}$ experiments of the Zürich group, at an optical lattice depth of 5 recoil energy for a laser wavelength of $825 \mathrm{~nm}$, a mean trapping frequency of $80 \mathrm{~Hz}$, and an (independently tuned) ratio $|U| / t=8$, the total number of fermions has to be smaller than $\sim 500$ to have a pure superfluid core. Remembering that this is only a rough estimate, this is nevertheless a rather small number to achieve under current conditions [40]. This critical number can be larger if we allow for a central region 
of band insulator in addition to a superfluid shell. Depending on the sensitivity to detect the superfluid shell, and thus the number of atoms that one needs to have in the superfluid, the number of fermions can be larger than the above estimate. The situation is better for the lighter atom ${ }^{6} \mathrm{Li}$. Using the same numbers as above, but with a laser wavelength of 1064 $\mathrm{nm}$, the upper critical number becomes $\sim 7000$, which should be feasible in current experiments.

As for experimental signature, the observation of the coherence peak in the momentum distribution should signify the onset of the BEC of pairs, and superfluidity can be proven when vortices are observed by rotating the trap and the optical lattice (although to date, vortices have been seen in a rotating bosonic BEC superfluid in an optical lattice [41]). These are simpler probes than, say, using noise correlation [42-44] to deduce the broken translation symmetry of the AFM state in the $U>0$ case [45].

\section{EFFECT OF DOPING}

So far, we have shown that the spin-balanced population for $U<0$ already presents several advantages to tackle the Mott and AFM physics. But one of its main advantages is the possibility to effectively "dope" the $U>0$ system by looking at spin imbalanced $U<0$ systems. This then allows us to settle experimentally the still controversial issue of the presence or absence of the $d$-wave superfluid $(d \mathrm{SF})$ in the repulsive Hubbard model doped away from half filling. As mentioned in Sec. I, this doping may be difficult to do directly in the $U>0$ system due to the presence of the overall harmonic trap. Via the transformation, the $U>0$ model away from half filling maps to the $U<0$ model with an effective Zeeman field. In the context of cold atom experiments, this corresponds to a (fixed) imbalance of spin-up versus spin-down fermions, which can readily be achieved to an accuracy of a few percent currently [46] (see [23] for a review).

We now examine some of the observables in that case, and in particular what would be the consequences of the existence of a $d$-wave superfluid phase in the repulsive Hubbard model for the $U<0$ phase. We perform this analysis for the homogeneous system and will discuss the possible effects of the trap at the end of this section.

\section{A. Transformation of the operators}

Under the transformation [Eq. (6)] the superfluid order parameter $\Delta^{(\alpha) \dagger}$ (the label $\alpha=s, d$ indicates the $s$-wave or $d$-wave symmetry of the order parameter) maps to the commensurate antiferromagnetic order parameter $M_{\text {stag }}^{(\alpha)+}$,

$$
\begin{aligned}
& \Delta^{(\alpha) \dagger}=\sum_{k} \phi_{k}^{(\alpha)}\left\langle c_{k \uparrow}^{\dagger} c_{-k \downarrow}^{\dagger}\right\rangle \leftrightarrow \sum_{k} \phi_{k}^{(\alpha)}\left\langle c_{k \uparrow}^{\dagger} c_{k+Q \downarrow}\right\rangle=M_{Q}^{(\alpha)+}, \\
& \Delta^{(\alpha)}=\sum_{k} \phi_{k}^{(\alpha)}\left\langle c_{-k \downarrow} c_{k \uparrow}\right\rangle \leftrightarrow \sum_{k} \phi_{k}^{(\alpha)}\left\langle c_{k+Q \downarrow}^{\dagger} c_{k \uparrow}\right\rangle=M_{Q}^{(\alpha)-},
\end{aligned}
$$

where previously, $\phi_{k}^{(s)}=1$ is the $s$-wave form factor and $\phi_{k}^{(d)}=\left(\cos k_{x}-\cos k_{y}\right)$ is the $d_{x^{2}-y^{2}}$ form factor.

Thus, if there is a regime of $d S F$ in the $U>0$ Hubbard model, then correspondingly, there is a regime of $d A F M$ in the $U<0$ model. This is the analog of the well-known mapping at precisely half filling of the Hubbard model between the ground states of $s \mathrm{SF}$ or $s \mathrm{CDW}$ at $U<0$ and the $s \mathrm{AFM}$ at $U>0$ (see Fig. 1 and Table I).

\section{B. Momentum distribution and noise correlation: A mean-field calculation}

In this section, we briefly outline a simple calculation to illustrate experimental signatures of $d \mathrm{AFM}$ states that may exist in the $U<0$ system. We need to emphasize from the outset that since there is no microscopic analytical calculation of the $d \mathrm{SF}$ nor the $d \mathrm{AFM}$ state in the 2D Hubbard model, we will instead use a toy mean-field (MF) model that does give rise to such states, in order to calculate some expected responses such as the momentum distribution and noise correlation [42-44]. While such a mean-field model misses out correlations and quantum fluctuations, the objective here is to demonstrate that the symmetry of the CDW or $S F$ order parameter has very definite signatures in noise correlation experiments [45]. Thus, we simply assume that the $U<0$ Hubbard model acquires a MF form, which in momentum space becomes

$$
\begin{aligned}
H_{\mathrm{MF}}^{(\alpha)}= & \sum_{k}\left[\left(\epsilon_{k \sigma}\right) c_{k \sigma}^{\dagger} c_{k \sigma}-h \sum_{\sigma} \sigma c_{k \sigma}^{\dagger} c_{k \sigma}\right]+\sum_{k} g_{Q} \phi_{k}^{(\alpha)} \\
& \times\left(c_{k \uparrow}^{\dagger} c_{k+Q \downarrow} M_{Q}^{(\alpha)-}+M_{Q}^{(\alpha)+} c_{k+Q \downarrow}^{\dagger} c_{k \uparrow}\right) .
\end{aligned}
$$

where we have inserted (by hand) the mean-field order parameter (12), and as before $\alpha$ labels the $s$ or $d_{x^{2}-y^{2}}$ order parameter. As usual, via a global gauge transformation, the order parameter can be chosen to be real: $M_{Q}^{(\alpha)-}=M_{Q}^{(\alpha)+}$ $=M_{Q}$. Our main interest is in the phases of the $U>0$ Hubbard mode without Zeeman field away from half filling. This then corresponds to the $U<0$ mean-field model above [Eq. (12)] at $\mu=0$ and finite $h$. In principle, the nesting wave vector $Q$ should be a variational parameter to be determined from the particular band structure. However, the following mean-field theory only makes sense if $Q=(\pi / a, \pi / a)$ is a commensurate wave vector: the interaction above couples $k$ with $k+Q$, and this in turn couples to $k+2 Q$ which is the same as $k$ only for commensurate $Q$.

This MF Hamiltonian is diagonalized by a Bogoliubov rotation as follows:

$$
\begin{gathered}
\alpha_{k}^{\dagger}=\cos \left(\theta_{k}\right) c_{k \uparrow}^{\dagger}+\sin \left(\theta_{k}\right) c_{k+Q \downarrow}^{\dagger}, \\
\beta_{k}^{\dagger}=-\sin \left(\theta_{k}\right) c_{k \uparrow}^{\dagger}+\cos \left(\theta_{k}\right) c_{k+Q \downarrow}^{\dagger} .
\end{gathered}
$$

Then, provided

$$
\begin{gathered}
\cos \left(\theta_{k}\right)=\left[\frac{1}{2}\left(1+\frac{\epsilon_{k \uparrow}-\epsilon_{k+Q \downarrow}-2 h}{2 \Omega_{k}}\right)\right]^{1 / 2}, \\
\sin \left(\theta_{k}\right)=\left[\frac{1}{2}\left(1-\frac{\epsilon_{k \uparrow}-\epsilon_{k+Q \downarrow}-2 h}{2 \Omega_{k}}\right)\right]^{1 / 2}, \\
\Omega_{k}=\left[\left(g_{Q} M_{Q} \phi_{k}\right)^{2}+\left(\frac{\epsilon_{k \uparrow}-\epsilon_{k+Q \downarrow}-2 h}{2}\right)^{2}\right]^{1 / 2},
\end{gathered}
$$

the MF Hamiltonian becomes 


$$
H_{\mathrm{MF}}=\sum_{k}\left(E_{k}^{\alpha} \alpha_{k}^{\dagger} \alpha_{k}+E_{k}^{\beta} \beta_{k}^{\dagger} \beta_{k}\right)
$$

with the "magnetic band" energies

$$
\begin{aligned}
& E_{k}^{\alpha}=\frac{\epsilon_{k \uparrow}+\epsilon_{k+Q \downarrow}}{2}+\Omega_{k}, \\
& E_{k}^{\beta}=\frac{\epsilon_{k \uparrow}+\epsilon_{k+Q \downarrow}}{2}-\Omega_{k} .
\end{aligned}
$$

The ground state is then made up by filling these bands up to the respective Fermi surface $k_{\alpha \sigma}^{F}$ with the occupation number $n_{k}^{\alpha}=\left\langle\mathrm{AFM}_{Q}\left|\alpha_{k}^{\dagger} \alpha_{k}\right| \mathrm{AFM}_{\mathbf{Q}}\right\rangle=\Theta\left(k_{\alpha}^{F}-k\right)$ (and similarly for the $\beta$ band),

$$
\left|\mathrm{AFM}_{Q}\right\rangle=\prod_{\sigma k} n_{k}^{\alpha} \alpha_{k}^{\dagger} n_{k}^{\beta} \beta_{k}^{\dagger}|0\rangle .
$$

Note that this wave function does not have definite numbers $N_{\uparrow}$ and $N_{\downarrow}$ for each species: this is a consequence of the quasiparticles $\alpha$ and $\beta$ carrying indefinite spin, an analog of the textbook number nonconserving Bogoliubov quasiparticles or the BCS wave function or the BCS mean-field Hamiltonian. In turn, here for the AFM, we have indefinite spin (but definite charge) quasiparticles because we have assumed the mean-field decoupling to be in the $S_{+}$axis in spin space. It is straightforward to show (at least when $h=0$ ) that the same results can be gotten for a spin-conserving mean-field wave function (e.g., when the mean-field decoupling is in the $z$-spin axis).

On the square lattice, since $\epsilon_{k \sigma}+\epsilon_{k+Q \sigma}=-2 \mu_{\sigma}$, taking the same chemical potential and bare dispersion for the two spin species, $E_{k}^{\alpha, \beta}=-\mu \pm \Omega_{k}$. Hence for the $s$-wave case there is always a band gap of size $\geq 2 g_{Q} M_{Q}$ and the minimum gap occurs at $\epsilon_{k \uparrow}-\epsilon_{k+Q \downarrow}=2 h$. Thus the half-filled lattice (one fermion per site) with $N_{\uparrow}=N_{\downarrow}$ has the $\beta$ band completely filled up and an empty $\alpha$ band: this is a magnetic insulator since adding one more $\uparrow$ fermion has $c_{k \uparrow}^{\dagger} \rightarrow \cos \left(\theta_{k}\right) \alpha_{k}^{\dagger}$ creating an $\alpha$ particle, but this costs at least the band-gap energy (and simi-

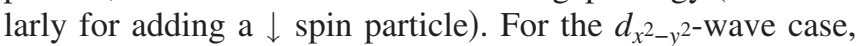
since the form factor $\phi_{k}=\cos k_{x}-\cos k_{y}$ has nodes at $k_{x}$ $= \pm k_{y}$, the band gap also vanishes at these positions, giving rise to a pseudogap only. At less-than-half filling (more-thanhalf filling), the "magnetic Fermi surface" is within the $\beta(\alpha)$ band and there is no energy gap to adding an extra fermion: the system is metallic. We should be careful about the meaning of $E_{k}^{\alpha, \beta}$ : this is not the physical excitation energy (unlike in the BCS MF theory); in particular, the spin-wave (Goldstone mode) spectrum is missing.

Using the ground state (16), the $T=0$ momentum distribution for each spin component is

$$
\begin{gathered}
\left\langle c_{k \uparrow}^{\dagger} c_{k \uparrow}\right\rangle=\cos ^{2}\left(\theta_{k}\right) n_{k}^{\alpha}+\sin ^{2}\left(\theta_{k}\right) n_{k}^{\beta}, \\
\left\langle c_{k+Q \downarrow}^{\dagger} c_{k+Q \downarrow}\right\rangle=\sin ^{2}\left(\theta_{k}\right) n_{k}^{\alpha}+\cos ^{2}\left(\theta_{k}\right) n_{k}^{\beta} .
\end{gathered}
$$

In experiments, assuming we can image perpendicular to the plane of the lattice, this result has to be convolved with the Wannier function $w(k)$ (in momentum space) for the optical lattice: $\left\langle n_{k=m R / \tau}^{\sigma}\right\rangle \propto|w(k)|^{2}\left\langle c_{k \sigma}^{\dagger} c_{k \sigma}\right\rangle$. (When there is more than

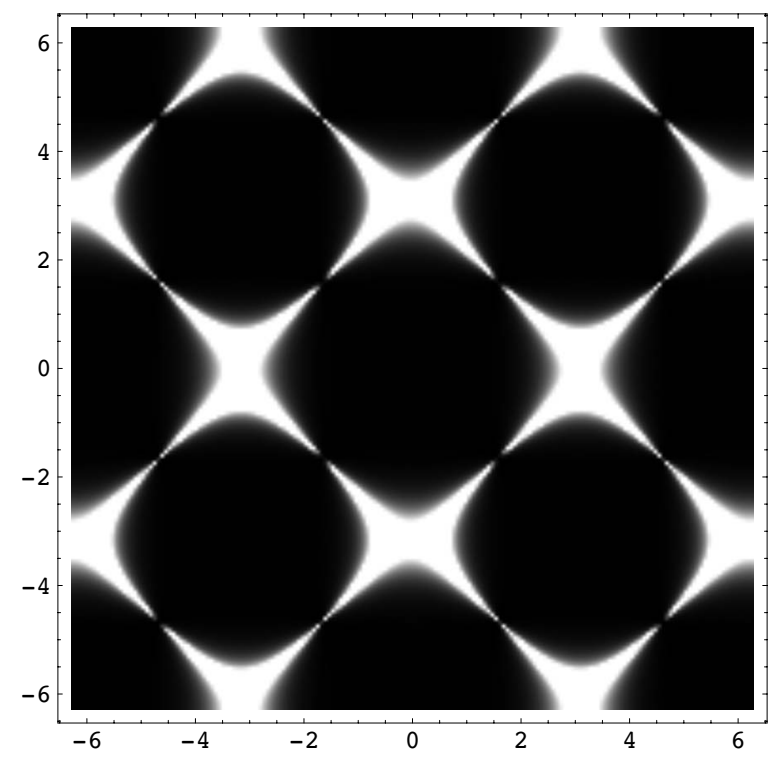

FIG. 5. Density (grayscale) plot of the noise correlation for the 2D $d_{x^{2}-y^{2}}$-wave AFM against $k_{x}$ and $k_{y}$ when $k^{\prime}=k+Q$. Parameters used: $g_{Q} M_{Q}=0.10, \mu=0$, and $h=0.23$.

one plane of the lattice, we also need to integrate over the planes.)

The $T=0$ noise correlation is proportional to the connected correlation function,

$$
\begin{aligned}
G_{\sigma \sigma^{\prime}}\left(k, k^{\prime}\right)= & \left\langle c_{k \sigma}^{\dagger} c_{k \sigma} c_{k^{\prime} \sigma^{\prime}}^{\dagger} c_{k^{\prime} \sigma^{\prime}}\right\rangle-\left\langle c_{k \sigma}^{\dagger} c_{k \sigma}\right\rangle\left\langle c_{k^{\prime} \sigma^{\prime}}^{\dagger} c_{k^{\prime} \sigma^{\prime}}\right\rangle \\
= & -\delta_{\sigma,-\sigma^{\prime}} \delta_{k^{\prime}, k+Q} \sin ^{2} \theta_{k} \cos ^{2} \theta_{k}\left[n_{k}^{\beta}\left(1-n_{k}^{\alpha}\right)\right] \\
= & \frac{-\delta_{\sigma,-\sigma^{\prime}} \delta_{k^{\prime}, k+Q}\left(g_{Q} M_{Q} \phi_{k}\right)^{2}}{4\left[\left(g_{Q} M_{Q} \phi_{k}\right)^{2}+\left(\frac{\epsilon_{k \uparrow}-\epsilon_{k+Q \downarrow}-2 h}{2}\right)^{2}\right]} \\
& \times\left[n_{k}^{\beta}\left(1-n_{k}^{\alpha}\right)\right] .
\end{aligned}
$$

(Again, for the experimentally measured noise correlation, this result has to be multiplied by $|w(k)|^{2}\left|w\left(k^{\prime}\right)\right|^{2}$.) We plot in

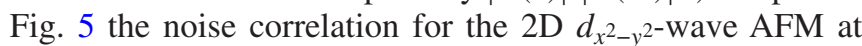
$\mu=0$. Noise correlation should clearly distinguish between $s$-wave and $d_{x^{2}-y^{2}} \mathrm{AFM}$; thanks to the appearance of nodes in the $\pm(\pi / a, \pi / a)$ directions.

\section{Experimental observation and complications}

We thus see that the negative- $U$ side offers the great advantage to access directly the doped regime, without having to suffer directly from the presence of the trap. We have presented in Sec. VI B some possible experimental signatures that a $d$-wave superfluid phase would give when suitably transformed to the negative side. Of course, even if the situation is potentially improved by the transformation to the negative- $U$ side, probing the doped phase is a considerable challenge. Some of the limitations are obvious. The most immediate one is the effect of the temperature. Indeed, already for the antiferromagnetic phase, the temperature is in competition with an energy on the order of $J \simeq 4 t^{2} / U$, the kinetic energy of the pairs. When looking at the doping ef- 
fects one has to face even smaller energies. Lowering the temperature is thus a must.

The second limitation is again the trap. Although the mapping to the negative side avoids the direct effect of the trap on the doped holes, the trap still has a potentially indirect effect. Indeed as we saw in the section on antiferromagnetism, the trap will act, for the $U>0$ side (model R1), as an effective Zeeman field and lead to two shell regions, with fully polarized spins up or down (Fig. 4). When holes are introduced into the system of model R1, they will thus have the possibility to go in one of these two polarized regions or in the antiferromagnetic region, which corresponds to the shell where the effective Zeeman field is not strong enough to fully polarize the system. Where the holes go will depend on how much kinetic and interaction energies they can gain in the three different phases since they are not sensitive directly to the presence of the Zeeman field. A naive calculation neglecting the presence of the energy scale $J$ leads directly to the holes going in the interface between the fully polarized up and fully polarized down regions. In the $U<0$ language, i.e., for model $\mathrm{A} 1$, this corresponds to the excess of one spin species going to the edge of the band insulator region. This seems to suggest that when the energy scale $J$ is put back in the problem, the holes will indeed go into the antiferromagnetic region and thus can lead potentially to the $d$-wave superconducting phase there. This is however a delicate question since the kinetic energy of a hole in a ferromagnetic environment is in principle higher due to the absence of frustration of the antiferromagnetic order upon hole motion. This important question thus fully deserves more analytical and numerical studies. It remains however academic until serious progress on the temperature issue has been made.

\section{SUMMARY AND CONCLUSION}

In this paper, we have explored in depth the possibilities offered by quantum simulating the attractive model (A1) in cold atoms in optical lattices to understand the physics of the repulsive Hubbard model, via a well-known canonical particle-hole transformation. We have argued that there are certain advantages in doing experiments in the attractive regime.
For the undoped case the attractive side replaces the Mott phase and the antiferromagnetic phase by a phase composed only of pairs (Mott insulator for $U>0$ ) and that undergoes a superfluid transition (antiferromagnet for $U>0$ ). The trap which exists in any realistic experiment does not really affect the observation of these two phases since it can only add a core of band insulator at the center, thereby not spoiling the observation of the superfluid. The attractive side also offers the advantage of only having to test for the pairing for the observation of the band insulator that must be simpler than testing for the absence of doubly occupied and empty sites on the repulsive side.

Another key advantage of the attractive side is the relative ease in controlling the spin population imbalance. Via the canonical transformation, this corresponds to doping away from half filling for the repulsive side, which is hard to achieve because of the presence of the harmonic trapping potential that moves the holes away from the central region of the trap. This doping in the repulsive regime is needed to quantum simulate and explore the possibility of a $d$-wave superfluid, the fundamental question of great relevance to the cuprate high-temperature superconductors. This question can be answered instead in the attractive regime by exploring the presence or absence of a $d$-wave antiferromagnet. We indicate in this paper several ways to probe for the existence of such a phase. We have also pointed out a number of technical difficulties of the proposed approach and, in some cases, suggest possible solutions.

\section{ACKNOWLEDGMENTS}

T.G. would like to thank M. Zwierlein for interesting discussions. We would like to thank H. Moritz for bringing to our attention an inaccurate estimate in Sec. V and for useful correspondence on the current experimental feasibility. M.A.C. thanks M. Ueda for his kind hospitality during his visit to the University of Tokyo and the Ueda Macroscopic Quantum Control Project of JST for partial financial support for this visit, during which parts of this work were done. This work was supported in part by the Swiss NSF under MaNEP and Division II. A.F.H. acknowledges financial support from EPSRC (UK) through Grant No. EP/D070082/1. M.A.C. gratefully acknowledges financial support of the Spanish MEC through Grant No. FIS2007-066711-C02-02 and CSIC through Grant No. PIE 200760/007.
[1] P. W. Anderson, Science 235, 1196 (1987).

[2] P. A. Lee, N. Nagaosa, and X.-G. Wen, Rev. Mod. Phys. 78, 17 (2004).

[3] L. Balents and M. P. A. Fisher, Phys. Rev. B 53, 12133 (1996).

[4] H. J. Schulz, Phys. Rev. B 53, R2959 (1996).

[5] E. Dagotto and T. M. Rice, Science 271, 618 (1996).

[6] T. Giamarchi, Quantum Physics in One Dimension (Oxford University Press, Oxford, UK, 2004).

[7] E. Demler, W. Hanke, and S.-C. Zhang, Rev. Mod. Phys. 76, 909 (2004)

[8] See, e.g., for a recent survey J. Zaanen, S. Chakravarty, T.
Senthil, P. Anderson, P. Lee, J. Schmalinn, M. Imada, D. Pines, M. Randeria, C. Varma, M. Vojta, and M. Rice, Nat. Phys. 2, 138 (2006).

[9] I. Bloch, J. Dalibard, and W. Zwerger, Rev. Mod. Phys. 80, 885 (2008).

[10] R. Jördens, N. Strohmaier, K. Günter, H. Moritz, and T. Esslinger, Nature (London) 455, 204 (2008).

[11] U. Schneider, L. Hackermüller, S. Will, Th. Best, I. Gloch, T. A. Costi, R. W. Helmes, D. Rasch, and A. Rosch, Science 322, 1520 (2008).

[12] See, e.g., the News Focus article by A. Cho, Science 320, 312 
(2008).

[13] A. M. Dare, L. Raymond, G. Albinet, and A. M. S. Tremblay, Phys. Rev. B 76, 064402 (2007); T. L. Ho and Q. Zhou, Phys. Rev. Lett. 99, 120404 (2007); T. L. Ho and Q. Zhou, e-print arXiv:0808.2652; T.-L. Ho, e-print arXiv:0808.2677.

[14] See, e. g., H.-J. Miesner, D. M. Stamper-Kurn, M. R. Andrews, D. S. Durfee, S. Inouye, and W. Kotare, Science 279, 1005 (1998); only recently sub-Poissonian number fluctuations have been achieved experimentally under special trapping conditions and using small number of atoms; see C.-S. Chuu, F. Schreck, T. P. Meyrath, J. L. Hanssen, G. N. Price, and M. G. Raizen, Phys. Rev. Lett. 95, 260403 (2005); taking into account total number fluctuations is also important for quantum simulation schemes based on finite-size scaling; see V. L. Campo, Jr., K. Capelle, J. Quintanilla, and C. Hooley, ibid. 99, 240403 (2007).

[15] C. Chin, R. Grimm, P. Julienne, and E. Tiesinga, e-print arXiv:0812.1496.

[16] R. B. Diener and T.-L. Ho, Phys. Rev. Lett. 96, 010402 (2006).

[17] A. F. Ho, Phys. Rev. A 73, 061601(R) (2006).

[18] F. Werner, O. Parcollet, A. Georges, and S. R. Hassan, Phys. Rev. Lett. 95, 056401 (2005)

[19] A. Moreo and D. J. Scalapino, Phys. Rev. Lett. 98, 216402 (2007).

[20] Andreas Lüscher, R. M. Noack, and A. M. Läuchli, Phys. Rev. A 78, 013637 (2008).

[21] M. Keller, W. Metzner, and U. Schollwöck, Phys. Rev. Lett. 86, 4612 (2001); M. Capone, C. Castellani, and M. Grilli, ibid. 88, 126403 (2002); N. Dupuis, Phys. Rev. B 70, 134502 (2004).

[22] A. Toschi, P. Barone, M. Capone, and C. Castellani, New J. Phys. 7, 7 (2005); A. Toschi, M. Capone, and C. Castellani, Phys. Rev. B 72, 235118 (2005); A. Garg, H. R. Krishnamurthy, and M. Randeria, ibid. 72, 024517 (2005); T.-L. Dao, M. Ferrero, A. Georges, M. Capone, and O. Parcollet, Phys. Rev. Lett. 101, 236405 (2008); T. L. Dao, A. Georges, and M. Capone, Phys. Rev. B 76, 104517 (2007); A. E. Feiguin and F. Heidrich-Meisner, ibid. 76, 220508(R) (2007); M. Tezuka and M. Ueda, Phys. Rev. Lett. 100, 110403 (2008); M. Rizzi, M. Polini, M. A. Cazalilla, M. R. Bakhtiari, M. P. Tosi, and R. Fazio, Phys. Rev. B 77, 245105 (2008).

[23] W. Ketterle and M. Zwierlein, in Ultracold Fermi Gases, Proceedings of the International School of Physics "Enrico Fermi," Varenna, 2006, Course CLXIV, edited by M. Inguscio, W. Ketterle, and C. Salomon (IOS, Amsterdam, 2008).

[24] We wish to point out for the solid-state community reader that the effect of the physical magnetic field is purely to change the Zeeman energy of the hyperfine states of the two species of fermions considered here. In particular, since cold atoms are neutral, there are no orbital effects on the superfluid, such as the Meissner effect for a superconductor. Henceforth, we shall call the physical magnetic field the Zeeman field to emphasize this.

[25] Note that here because the interacting term contains a term proportional to $N$, the definition of $\mu$ is somewhat nonstandard.

[26] C. A. Regal, M. Greiner, and D. S. Jin, Phys. Rev. Lett. 92, 040403 (2004); M. W. Zwierlein, C. A. Stan, C. H. Schunck, S. M. F. Raupach, A. J. Kerman, and W. Ketterle, ibid. 92,
120403 (2004); C. A. Regal and D. S. Jin, ibid. 90, 230404 (2003).

[27] P. O. Fedichev, M. J. Bijlsma, and P. Zoller, Phys. Rev. Lett. 92, 080401 (2004).

[28] M. Wouters and G. Orso, Phys. Rev. A 73, 012707 (2006).

[29] M. A. Cazalilla, A. F. Ho, and T. Giamarchi (unpublished)

[30] By pairs it is meant in this paper a many-body bound state of the same nature as a Cooper pair. These pairs should be distinguished from molecular bound states arising from poles in the scattering amplitude of the two-body problem.

[31] C. Ryu, X. Du, E. Yesilada, A. M. Dudarev, S. Wan, Q. Niu, and D. J. Heinzen, e-print arXiv:cond-mat/0508201.

[32] T.-L. Dao, A. Georges, J. Dalibard, C. Salomon, and I. Carusotto, Phys. Rev. Lett. 98, 240402 (2007).

[33] J. T. Stewart, J. P. Gaebler, and D. S. Jin, Nature (London) 454, 744 (2008).

[34] G. K. Campbell, J. Mun, M. Boyd, P. Medley, A. E. Leanhardt, L. G. Marcassa, D. E. Pritchard, and W. Ketterle, Science 313, 649 (2006).

[35] S. Folling, A. Widera, T. Muller, F. Gerbier, and I. Bloch, Phys. Rev. Lett. 97, 060403 (2006).

[36] In the large $U / t$ limit, we can ignore the kinetic energy, and hence the Hamiltonian is purely local. The density profile is then $\left\langle n_{i \uparrow}+n_{i \downarrow}\right\rangle=2\left[\exp \beta \mu_{i}+\exp \beta\left(2 \mu_{i}-s|U|\right)\right] /\left[1+2 \exp \beta \mu_{i}\right.$ $\left.+\exp \beta\left(2 \mu_{i}-s|U|\right)\right]$, where $\mu_{i}=\mu-\epsilon_{i}+s|U| / 2$ and $s=-1$ for the attractive model A1 at $h=0$ and $s=+1$ for the repulsive model R2. The total number of fermions $N=\Sigma_{i}\left\langle n_{i \uparrow}+n_{i \downarrow}\right\rangle$, which fixes the chemical potential $\mu(T, U)$. We have also found that, at low temperatures, the density profiles for $U<0$ can be obtained from a mapping to a free fermion model; details of this model and its consequences will be reported elsewhere [29].

[37] See, e.g., A. Auerbach, Interacting Electrons and Quantum Magnetism (Springer-Verlag, Berlin, 1990).

[38] Another way to see the same correspondence is using wave functions. A cartoon ground state for AFM ordering in the $x$ direction is of the form $\left|\mathrm{AFM}_{x}\right\rangle \sim \Pi_{j}\left[c_{j \uparrow}^{\dagger}+(-1)^{j} c_{j \downarrow}^{\dagger}\right]\left|\operatorname{vac}_{c}\right\rangle$, where $\left|\operatorname{vac}_{c}\right\rangle$ is the vacuum state for the $c$ fermions. Defining $d_{j \uparrow}^{\dagger}=c_{j \uparrow}^{\dagger} \quad$ and $\quad d_{j \downarrow}=(-1)^{j} c_{j \downarrow}^{\dagger} \quad$ with $\quad\left|\operatorname{vac}_{c}\right\rangle=\Pi_{j} d_{j \downarrow}^{\dagger}\left|\operatorname{vac}_{d}\right\rangle$, then $\left|\mathrm{AFM}_{x}\right\rangle \rightarrow \Pi_{j}\left[d_{j \uparrow}^{\dagger} d_{j \downarrow}^{\dagger}+1\right]\left|\operatorname{vac}_{d}\right\rangle$, which is a coherent state of pairs $b_{j}^{\dagger}=d_{j \uparrow}^{\dagger} d_{j \downarrow}^{\dagger}$.

[39] Note that for small $U$ the interplay between the inhomogeneous magnetic field and the kinetic energy leads to interesting effects for the intermediate phase due to the difference between the radial and angular directions for the hopping of a particle. These effects will be discussed elsewhere [29].

[40] H. Moritz (private communication).

[41] S. Tung, V. Schweikhard, and E. A. Cornell Phys, Rev. Lett. 97, 240402 (2006).

[42] S. Fölling, F. Gerbier, A. Widera, O. Mandel, T. Gericke, and I. Bloch, Nature (London) 434, 481 (2005).

[43] T. Rom, Th. Best, D. van Ooster, U. Schneider, S. Fölling, and B. Paredes, and I. Bloch, Nature (London) 444, 733 (2006).

[44] M. Greiner, C. A. Regal, J. T. Stewart, and D. S. Jin, Phys. Rev. Lett. 94, 110401 (2005).

[45] E. Altman, E. Demler, and M. D. Lukin, Phys. Rev. A 70, 013603 (2004).

[46] M. Kohl, H. Moritz, T. Stoferle, K. Gunter, and T. Esslinger, Phys. Rev. Lett. 94, 080403 (2005). 